\title{
Actividad insecticida de aceites esenciales sobre Helicoverpa armígera (Hübner) (Lepidoptera: Noctuidae)
}

\author{
Essential oils insecticide activity on Helicoverpa armigera \\ (Hübner) (Lepidoptera: Noctuidae) \\ Cristhian Eliseo Durán Aguirre ${ }^{1}$, Dirceu Pratissoli ${ }^{1}$, José Romário de Carvalho', \\ Alixelhe Pacheco Damascena ${ }^{1 *}$, Luis Moreira de Araujo Junior ${ }^{1}$, Hugo Bolsoni Zago ${ }^{1}$
}

\section{RESUMEN}

El objetivo de este estudio fue evaluar la actividad insecticida de los aceites esenciales Citrus aurantium (Linnaeus) (Rutaceae), Citrus sinensis (Linnaeus) (Rutaceae) y el componente mayoritario Limoneno, y aceites esenciales Piper spp. (Linnaeus) (Piperaceae), Syzygium aromaticum (Merrill \& Perry) (Myrteraceae), Cinnamomum zeylanicum (Presl) (Lauraceae), Zingiber officinale (Roscoe) (Zingiberaceae) y Rosmarinus officinalis (Linnaeus) (Lamiaceae) sobre larvas de primer instar de H. armígera. La extracción de los aceites esenciales se realizó mediante la técnica de hidrodestilación utilizando el Clevenger modificado. Se proporcionaron $2 \mathrm{ml}$ de la solución de cada aceite esencial a una concentración del $2 \%\left(\mathrm{v} \cdot \mathrm{v}^{-1}\right)$ sobre larvas de primer instar de H. armígera, utilizando un aerógrafo con presión de $15 \mathrm{lb} / \mathrm{in}^{2}$. Se realizaron diez réplicas por cada tratamiento, con diez larvas por cada repetición. Se evaluó la toxicidad aguda de los aceites esenciales mediante el recuento de larvas muertas después de 48 horas de ser tratadas. Los aceites esenciales $C$. aurantium, el componente mayoritario Limoneno, C. sinensis, $R$. officinalis, C. zeylanicum, S. aromaticum, Z. officinale y Piper spp. mostraron mortalidad de 97, 94, 87, 9, 8, 3, 2 y 0\%, respectivamente. Los aceites esenciales del género Citrus spp. presentaron un alto porcentaje de mortalidad, y son una alternativa para el desarrollo de nuevas moléculas activas a partir de compuestos orgánicos, que pueden incorporarse dentro de un programa de control biológico para H. armígera.

Palabras clave: metabolitos secundarios, alelo-químicos, insecticidas botánicos.

\begin{abstract}
The objective was to evaluate the insecticidal activity of essential oils Citrus aurantium (Linnaeus) (Rutaceae), Citrus sinensis (Linnaeus) (Rutaceae) and the majoritary component Limonene; and essential oils Piper spp. (Linnaeus) (Piperaceae); Syzygium aromaticum (Merrill \& Perry) (Myrteraceae); Cinnamomum zeylanicum (Presl) (Lauraceae); Zingiber officinale (Roscoe) (Zingiberaceae) and Rosmarinus officinalis (Linnaeus) (Lamiaceae) about first instar larvae of $\mathrm{H}$. armigera. The extraction of essential oils was performed by hydrodistillation technique using the Clevenger modified. Provided $2 \mathrm{~mL}$ of the solution of each essential oil at $2 \%\left(v \cdot v^{-1}\right)$ concentration on first instar larvae of H. armigera, using an airbrush with pressure $15 \mathrm{lb} / \mathrm{in}^{2}$. Ten replicates were performed for each treatment, with 10 caterpillars per replicate. It was evaluated the acute toxicity of essential oils by dead caterpillars count after 48 hours treated. Essential oils C.aurantium, the majoritary component Limonene, C. sinensis, R. officinalis, C. zeylanicum, S. aromaticum, Z. officinale and Piper spp. showed mortality of 97, 94, 87, 9, 8, 3, 2 and $0 \%$ respectively. Essential oils of Citrus spp. genus were emphasized showing the highest percentage of mortality, being an alternative for developing new active molecules form organic compounds, which can be incorporated into a biological control program for $\mathrm{H}$. armigera.
\end{abstract}

Keywords: secundary metabolites, allelochemicals, botanical insecticides.

\section{Introducción}

Las plantas, como organismos que evolucionan con los insectos y microorganismos, pueden desarrollar mecanismos de comunicación debido a la constante presión ambiental y como defensa contra el ataque de insectos, hongos, bacterias, virus y entomopatógenos en la producción, y liberación de sustancias orgánicas como los aceites esenciales (Bakkali et al., 2008).

Estos aceites esenciales son una mezcla compleja de varios químicos aromáticos, con

\footnotetext{
1 Departamento de Agronomía, Centro de Ingeniería y Ciencias Agrarias, Universidade Federal do Espírito Santo, Alto Universitário, s/n, Guarerema, 29.500-000, Alegre, Espírito Santo, Brasil.

* Autor por correspondencia: xellydamascena@hotmail.com
}

Fecha de Recepción: 2 de Junio, 2020.

Fecha de Aceptación: 20 de Agosto, 2020. 
una densidad inferior a la del agua, soluble en lípidos y solventes orgánicos. Generalmente se producen a partir de los metabolitos secundarios de las plantas, que varían en concentración y composición de acuerdo a las especies, órganos y modo de extracción, época de recolección, condiciones climáticas y tipo de suelo (IonescuMălăncus et al., 2013).

En agricultura, se destaca la aplicabilidad de estos compuestos para el control de insectos plagas, a través de diferentes mecanismos de acción que afectan múltiples objetivos, alterando efectivamente la actividad celular y los procesos biológicos de las plantas (Simões et al., 2007; Santa-Cecília et al., 2010).

Muchos aceites esenciales tienen propiedades toxicológicas contra insectos de los órdenes coleóptera, lepidóptera, hemíptera, díptera, orthoptera phthiraptera y artrópoda isóptera, debido a que tienen múltiples modos de acción, incluida actividad repelente y antinutritiva, inhibición de la respiración, reducción del crecimiento y fertilidad, destrucción de la cutícula y actividad octopamínica en el sistema nervioso central (Yang et al., 2017; Ghabbari et al., 2018).

Los insecticidas botánicos poseen la ventaja de reducir el riesgo de resistencia cruzada por la compleja estructura química de los constituyentes, reduciendo así la presión de selección de insectos resistentes a pesticidas. Adicionalmente, la rápida degradación causa menos daños a la salud humana y al medio ambiente. Muchos aceites esenciales son específicos y ocasionan pocos daños a los organismos no objetivos y enemigos naturales al ser comparados con insecticidas sintéticos (Zacharia, 2011).

Helicoverpa armígera (Hübner) (Lepidoptera: Noctuidae) es un insecto responsable de causar graves daños a varios cultivos de gran importancia económica. El principal método de manejo de esta plaga es el control químico con pulverizaciones frecuentes de insecticidas durante el ciclo del cultivo, lo que desencadena efectos negativos para la salud humana y el medio ambiente, así como el desarrollo de resistencia en los insectos y el incremento de los costos de producción (Czepack et al., 2013).

El objetivo de este estudio fue evaluar la actividad insecticida de los aceites esenciales Citrus aurantium (Linnaeus) (Rutaceae), Citrus sinensis (Linnaeus) (Rutaceae) y el componente mayoritario Limoneno, y aceites esenciales Piper spp. (Linnaeus) (Piperaceae), Syzygium aromaticum (Merrill \& Perry) (Myrteraceae), Cinnamomum zeylanicum (Presl) (Lauraceae), Zingiber officinale (Roscoe) (Zingiberaceae) y Rosmarinus officinalis (Linnaeus) (Lamiaceae) sobre larvas de $H$. armígera, con la intención de buscar métodos alternativos de manejo que se puedan incorporar dentro de un programa de manejo integrado de plagas.

\section{Materiales y métodos}

La investigación se llevó a cabo en el Laboratorio del Núcleo de Desenvolvimento Científico e Tecnológico em Manejo Fitossanitário de Pragas e Doenças (NUDEMAFI), sector de Entomología, Departamento de Producción de Plantas y el Laboratorio de Química, Departamento de Química y Física en el Centro de Ingeniería y Ciencias Agrarias de la Universidade Federal do Espírito Santo (CCAE/UFES).

\section{Multiplicación y creación de Helicoverpa armigera}

Larvas de $H$. armígera utilizadas en el experimento provenían del stock del Laboratorio de Entomología del Núcleo de Desenvolvimento Científico e Tecnológico em Manejo Fitossanitário de Pragas e Doenças (NUDEMAFI), donde los adultos fueron colectados y colocados en tubos de PVC (20 cm de diámetro x $25 \mathrm{~cm}$ de altura) cubiertos internamente con papel blanco, el extremo superior cerrado con papel toalla y malla tul y el extremo inferior apoyado en una base de espuma cuadrada de poliestireno recubierta con papel blanco $(25 \mathrm{~cm}$ largo x $3 \mathrm{~cm}$ ancho). Se suministró una solución de miel al $10 \%\left(\mathrm{~m}^{-1}\right)$ a los adultos por medio de algodón embebido en un vial de vidrio de $5 \mathrm{ml}$.

Las posturas de huevos de $H$. armígera depositadas en las paredes internas de los tubos PVC y en papel blanco fueron colectadas y colocadas en placas de Petri (14 cm de diámetro x $15 \mathrm{~cm}$ de altura). Después de la eclosión, las larvas fueron individualizadas en tubos de vidrio $(8.5 \mathrm{~cm}$ de diámetro x $2.5 \mathrm{~cm}$ de altura) con dieta artificial con volumen de hasta $1 / 3$, a base de frijol, germen de trigo, harina de soya y levadura de cerveza (Grenne et al., 1976).

Las larvas estuvieron en los tubos de vidrio durante todo el período pupal. Luego fueron 
retiradas de dichos tubos, tratadas con hipoclorito de sodio al $10 \%$ y lavadas con agua desionizada. Todas las etapas de desarrollo de $H$. armígera fueron mantenidas en condiciones controladas con temperatura de $25 \pm 1{ }^{\circ} \mathrm{C}$, humedad relativa de $65 \pm 10 \%$ y fotoperiodo de 12 horas.

\section{Aceites esenciales}

Los aceites esenciales comerciales de naranja amarga (C. aurantium) y naranja dulce ( $C$. sinensis) fueron comprados en Ferquima Industria y Comercio Ltda y el componente mayoritario D-Limoneno en Sigma Aldrich Company.

Los materiales para la extracción de los aceites esenciales de hojas, frutas, rizomas y brotes florales de pimienta (Piper spp.), clavo indio (S. aromaticum), canela (C. zeylanicum), jengibre (Z. officinale) y romero (R. officinalis) se obtuvieron comercialmente en la ciudad de Alegre, Espírito Santo. Luego fueron cortados en pequeñas porciones de aproximadamente $300 \mathrm{~g}$ y sometidos a hidrodestilación por triplicado. Cada material se colocó en un matraz de 51 y se añadió agua destilada a la mitad del matraz que posteriormente fue acoplado al Clevenger y sometido a calentamiento. De esta forma, la hidrodestilación de los materiales se llevó a cabo por tres horas consecutivas (Pinheiro et al., 2013).

Después de la hidrodestilación, el hidrolato obtenido se sometió a extracción líquido-líquido con pentano $(3 \times 20 \mathrm{ml})$. Para remover el agua restante se añadió Na2SO4 (sulfato de sodio) anhidro en la fase orgánica y luego se realizó filtración. El material obtenido se concentró por evaporador rotatorio a presión reducida, verificándose la masa de los aceites para el cálculo de los rendimientos. Los aceites esenciales extraídos se envasaron en frasco de vidrio ámbar y se almacenaron en un congelador para ser analizados (Pinheiro et al., 2013).

En los bioensayos, se pulverizaron larvas de primer instar de $H$. armígera con $2 \mathrm{ml}$ de la solución de cada aceite esencial Citrus aurantium, Limonene, Citrus sinensis, Rosmarinus officinalis, Cinnamomum zeylanicum, Syzygium aromaticum, Zingiber officinale y Piper spp. a una concentración del $2 \%\left(\mathrm{v} . \mathrm{v}^{-1}\right)$ usando un aerógrafo con una presión de $15 \mathrm{lb} / \mathrm{in} 2$. Las larvas fueron separadas en grupos de 10 individuos en placas de Petri ( $9 \mathrm{~cm}$ diámetro x $1.5 \mathrm{~cm}$ altura) y colocándoles dieta artificial. Se realizaron diez repeticiones por cada tratamiento, con diez larvas por cada réplica. La toxicidad de los aceites esenciales se evaluó contando las larvas muertas después de 48 horas de ser tratadas.

\section{Análisis estadístico}

Los datos de mortalidad de $\mathrm{H}$. armígera fueron registrados 48 horas después de establecido el experimento, corregidos por la fórmula de Abbott y sometidos a análisis de varianza y comparación de medias por la prueba de Scott-Knott al 5\% de significancia por el programa SAS (SAS Institute, 2002).

\section{Resultados y discusión}

Los aceites esenciales de naranja amarga (C. aurantium), el componente mayoritario Limoneno y naranja dulce (C. sinensis) presentaron una mortalidad de 97,94 y $87 \%$ respectivamente, diferenciándose significativamente de los aceites esenciales de romero ( $R$. offinalis), canela (C. zeylanicum), clavo indio ( $S$. aromaticum), jengibre (Z. officinale) y pimienta (Piper spp.) con $9,8,3,2$ y $0 \%$ de mortalidad respectivamente, $\left.\mathrm{F}_{7,27}=390.27 ; \mathrm{P}<0.005\right)$ (Tabla 1).

La composición, concentración e interacción de los compuestos orgánicos de los aceites

Tabla 1. Mortalidad corregida de larva de primer instar de Helicoverpa armígera con aceites esenciales de Citrus aurantium, Limoneno, Citrus sinensis, Rosmarinus officinalis, Cinnamomum zeylanicum, Syzygium aromaticum, Zingiber officinale y Piper spp. por pulverización.

\begin{tabular}{lcc}
\hline Tratamiento $^{1}$ & $\mathrm{~N}^{2}$ & (\%) $^{\text {Mortalidad }}{ }^{3}$ \\
\hline Naranja amarga (Citrus aurantium) & 100 & $97 \mathrm{a}$ \\
Limoneno & 100 & $94 \mathrm{a}$ \\
Naranja dulce (Citrus sinensis) & 100 & $87 \mathrm{a}$ \\
Romero (Rosmarinus officinalis) & 100 & $9 \mathrm{~b}$ \\
Canela (Cinnamomum zeylanicum) & 100 & $8 \mathrm{~b}$ \\
Clavo indio (Syzygium aromaticum) & 100 & $3 \mathrm{~b}$ \\
Jengibre (Zingiber officinale) & 100 & $2 \mathrm{~b}$ \\
Pimienta (Piper spp.) & 100 & $0 \mathrm{~b}$ \\
CV $(\%)$ & & 19,58 \\
P & & $<0,005$ \\
\hline
\end{tabular}

Temperatura: $25 \pm 1{ }^{\circ} \mathrm{C}, \mathrm{HR}: 65 \pm 10 \%$ y fotoperiodo de $12 \mathrm{~h}$; 1 Medias seguidas de la misma letra en la columna no difieren estadísticamente por la prueba de Scott-Knott $(\mathrm{P}<0,05) ; 2 \mathrm{~N}$ : número de individuos por tratamiento; 3 (\%) Mortalidad corregida por la fórmula de Abbott. 
esenciales depende de la especie, órgano, modo de extracción, tiempo de cosecha, condiciones climáticas, tales como radiación, temperatura, humedad relativa, precipitación y fotoperíodo (Simões et al., 2007). Por lo tanto, la eficiencia de Citrus spp. en la mortalidad de H. armígera puede estar influenciada por la diferenciación en la composición, modo y condiciones de extracción de los aceites esenciales en estudio.

Naranja amarga, el componente mayoritario Limoneno y naranja dulce fueron seleccionados para los siguientes bioensayos, y son los aceites esenciales que presentaron mortalidad $\geq 80 \%$, porcentaje para que una molécula se considere eficiente en el control de plagas de acuerdo con el Ministerio de Agricultura, Ganadería y Abastecimiento (MAPA, 2014).

Los aceites esenciales de Citrus spp. vienen siendo estudiados debido a sus efectos insecticidas sobre artrópodos, actuando por ingestión, contacto o fumigación. Son considerados promisorios para el manejo integrado de plagas. Además de su acción insecticida, tienen efectos sobre el crecimiento, la reducción de la oviposición, repelencia y actividad ovicida. Estudios han demostrado la actividad insecticida de aceites esenciales de cítricos sobre Spodoptera litura (Fabricius) (Lepidoptera: Noctuidae) y Myzus persicae (Sulzer) (Hemiptera: Aphididae) (Brito et al., 2006).

Estudios han reportado que los monoterpenos limoneno, $\beta$-pineno, $\alpha$-pineno dañan y obstruyen las vías respiratorias de los insectos, actuando rápidamente e interfiriendo en las funciones fisiológicas por la adhesión de las partículas de los aceites esenciales en la cutícula de los espiráculos (Prates et al., 1998). Otros estudios muestran que los monoterpenos actúan como inhibidores de la acetilcolinesterasa, lo que lleva a un exceso del neurotransmisor acetilcolina y produce un colapso del espacio sináptico generalizado, provocando la muerte del insecto por asfixia (Tsukamoto et al., 2005).

Por otro lado, la baja mortalidad presentada por los aceites esenciales romero (R. offinalis), canela (C. zeylanicum), clavo indio (S. aromaticum), jengibre (Z. officinale) y pimienta (Piper spp.) sobre larvas de $H$. armígera podría estar vinculada a una relación antagónica entre sus compuestos mayoritarios y minoritarios. Además, se debe tener en cuenta que la composición, cantidad y concentración de los componentes de los aceites esenciales depende de factores como condiciones climáticas, edad de la planta y época de extracción. Las interacciones entre estos compuestos son complejas y pueden afectar las características fisicoquímicas de los aceites esenciales (Jiang et al., 2009).

En un estudio desarrollado por Born et al. (2009) se observó que el aceite esencial de romero (R. officinalis) presentó acción acaricida y redujo la fecundidad contra el ácaro rayado Tetranychus urticae (Koch) (Acari: Tetranychidae). Las concentraciones letales $\mathrm{CL}_{50}$ y $\mathrm{CL}_{90}$ del aceite esencial de $R$. offinalis sobre el ácaro rayado fueron 0,77 y $1,77 \mu \mathrm{L} / \mathrm{L}$, respectivamente. Otros trabajos realizados por Miresmailli e Isman (2006) registraron toxicidad del aceite de romero contra el ácaro rayado por contacto.

Los constituyentes mayoritarios del aceite esencial de canela $C$. zeylanicum son el eugenol, fenilpropanoides como cinamaldehido, acetato de cinamila y 1,8-cineol. Estudios han demostrado que los extractos de $C$. zeylanicum poseen propiedades fúngicas contra Cladosporium musae (Mason) (Capnodiales: Mycosphaerellaceae), Lasiodiplodia theobromae (Patouillard) (Botryosphaeriales: Botryosphaeriaceae) y Fusarium proliferatum (Matsush) (Hypocreales: Nectriaceae); actividad ectoparásita contra Pedaculus humanus capatis (De Geer) (Anoplura: Pediculidae) y acción acaricida contra Psoroptes cuniculi (Delafond) (Sarcoptiformes: Psoroptidae) (Ranasinghe et al., 2002; Yang et al., 2005; Andrade et al., 2012).

El aceite esencial de clavo indio $S$. aromaticum está constituido por los compuestos eugenol, $\beta$-cariofileno y acetato de eugenol, elementos que inhiben el crecimiento de hongos de los géneros Monilia, Botrytis y Mucor. En artrópodos, los aceites esenciales de $S$. aromaticum demostraron inhibición en la emergencia de adultos de Culex pipiens (Linnaeus) (Diptera: Culicidae). Recientemente se demostró que el eugenol es tóxico para la hormiga roja Solenopsis invicta (Buren) (Hymenoptera: Formicidae) (Ranasinghe et al., 2002; Kafle y Shih, 2013).

Otros trabajos desarrollados por Pereira et al. (2008) constataron que el aceite esencial de jengibre (Z. officinale) promovió la inhibición en el crecimiento de las bacterias Staphylococcus aureus (Rosenbach) (Bacillales: Staphylococcaceae) y Escherichia coli (Escherich) (Enterobacteriales: Enterobacteriaceae). El aceite esencial de 
Z. officinale presenta actividad larvicida y repelencia sobre Culex quinquefasciatus Say (Diptera: Culicidae) (Pushpanathan et al., 2008).

El aceite del género Piper spp. contiene una serie de amidas en la estructura química, y el mayor constituyente es la piperina, que actúa como neurotóxico afectando las principales funciones del sistema nervioso central de los insectos. Extractos cetónicos y metanólicos de Piper nigrum (Linnaeus) (Piperaceae) causaron $90 \%$ de la mortalidad de las larvas de los cereales Sitotroga cerealella (Oliver) (Lepidoptera: Gelechiidae) (Boff and Almeida, 1995). Paula et al., (2000, 2001) reportaron efectos insecticidas de piperina sobre el pulgón Brevicoryne brassicae (Linnaeus) (Hemiptera: Aphididae) y las termitas Cornitermes cumulans (Kollar) (Isoptera: Termitidae).

De lo expuesto se desprende que la actividad insecticida y los posibles efectos de los aceites esenciales dependen de la composición química, concentración e interacción de los constituyentes mayoritarios y minoritarios de estos compuestos orgánicos.

\section{Conclusiones}

Los aceites esenciales de $C$. aurantium, el componente mayoritario Limoneno, C. sinensis, $R$. officinalis, C. zeylanicum, S. aromaticum, Z. officinale y Piper spp. en la concentración de $2 \%$ (v. $\left.\mathrm{v}^{-1}\right)$ presentaron mortalidad de 97, 94, 87, 9, 8, 3, 2 y $0 \%$, respectivamente, sobre larvas de primer instar de $H$. armígera a las 48 horas de ser tratadas. Los aceites esenciales $C$. aromaticum, el componente mayoritario Limoneno y el $C$. sinensis mostraron los mayores porcentajes de mortalidad, por lo que futuros estudios podrían certificar la eficiencia de estos productos en campo, con el propósito de desarrollar una molécula activa para ser incorporada en el manejo integrado de $H$. armígera.

\section{Agradecimientos}

Este trabajo fue desarrollado con el apoyo de la Coordenação de Aperfeiçoamento de Pessoal de Nível Superior (CAPES) y la Fundação de Amparo à Pesquisa e Inovação do Espírito Santo (FAPES).

\section{Literatura Citada}

Andrade, M.A.; Cardoso-Graças, M.; Batista, L.R.; Mallet, A.C.T.; Machado, S.M.F.

2012. Óleos essenciais de Cymbopogom nardus, Cinnamomum zeylanicum e Zingiber officinale: composição, atividades antioxidante e antibacteriana. Revista Ciência Agronômica, 43 (2): 399-408.

Bakkali, F.; Averbeck, S.; Averbeck, D.; Idaomar, M. 2008. Biological effects of essential oils. Food and Chemical Toxicology, 46 (2): 446-475.

Brito, J.P.; Oliveira, J.E.M.; Bortoli, S.A.

2006. Toxicidade de óleos essenciais de Eucalyptus spp. sobre Callosobruchus maculatus (Fabr., 1775) (Coleoptera: Bruchidae). Revista de Biologia e Ciência da Terra, 6 (1): 96-103.

Boff, M.I.; Almeida, A.D. 1995. Efeito residual de extratos de Piper nigrum (L.) sobre larvas neonatas de Sitotroga cerealella (Oliv.). Anais da Sociedade Entomológica do Brasil, 24 (1): 115-121.

Born, F.S.; Ribeiro, N.C.; De Araújo, M.J.C.; Botelho, P.S.; De Moraes, M.M.; Neves, I.A.; Da Câmara, C.A.G.

2009. Atividade acaricida do óleo essencial de folhas de Rosmarinus officinalis (L.) sobre Tetranychus urticae (Koch) (Acari: Tetranychidae) resumo. Disponible en: <http://www.eventosufrpe.com.br/jepex2009/cd/resumos/ R0787-1.pdf>. Consultado: 01/jan/ 2020.

Czepak, C.; Albernaz, K.C.; Vivan, L.M.; Guimarães, H.O.; Carvalhais, $\mathrm{T}$.

2013. Primeiro registro de ocorrência de Helicoverpa armigera (Hübner) (Lepidoptera: Noctuidae) no Brasil. Pesquisa Agropecuária Tropical, 43 (1), 110-113.
Ghabbari, M.; Guarino, S.; Caleca, V.; Saiano, F.; Sinacori, M.; Baser, N.; Mediouni, J.; Verde, G.

2018. Behavior-modifying and insecticidal effects of plant extracts on adults of Ceratitis capitata (Wiedemann) (Diptera Tephritidae). Journal of Pest Science, 91 (1): 907-917.

Greene, G.; Leppla, N.C.; Dickerson, W.

1976. Velvet bean caterpillar: a rearing procedure and artificial medium. Journal of Economic Entomology, 69(4): 488-497.

Ionescu-Mălăncus, I.; Manole, T.; Niculiţă, P.; Petrescu, E.

2013. Possibilities to use natural extracts from medicinal and aromatic plants (map) like botanical repellent or insecticide compounds against pest insects in ecological crops (II). Scientific Papers Series Management, Economic Engineering in Agriculture and Rural Development, 13 (4): 173-178.

Jiang, Z., Akhtar, Y.; Bradbury, R.; Zhang, X.; Isman, M.B. 2009. Comparative toxicity of essential oils of Litsea pungens and Litsea cubeba and blends of their major constituents against the cabbage looper, Trichoplusiani. Journal of Agricultural and Food Chemistry, 57 (1): 4833-4837.

Kafle, L.; Shih, C.H.J.

2013. Toxicity and Repellency of Compounds from Clove (Syzygium aromaticum) to Red Imported Fire Ants Solenopsis invicta (Hymenoptera: Formicidae). Bio One Research Evolved, 106 (1): 131-135.

MAPA.

2014. Combate a praga Helicoverpa armigera. Ministério da Agricultura, Pecuária e Abastecimento. Brasília, Brasil. 4 p. 
Miresmailli, S.; Isman, M.B.

2006. Efficacy and Persistence of Rosemary Oil as an Acaricide Against Twospotted Sider Mite (Acari: Tetranychidae) on Greenhouse Tomato. Journal of Economic Entomolology, 99 (1): 2015-2023.

Paula, V.F.; Barbosa, L.C.A.; Picanço, M.C.; Pilóveloso, D. 2001. Toxicidade de amidas derivadas da piperina para larvas de Ascia monusteorseis (Godart) (Lepdoptera: Pieridae). Revista Brasileira de Entomologia, 45 (1): 10-15.

Paula, V.F.; Barbosa, L.C.A.; Demuner, A.J.; Pilóveloso, D.; Picanço, M.C.

2000. Synthesis and insecticidal activity of new amide derivates of piperines. Pest Management Science, 56 (1): 168-174.

Pereira, A.A.; Cardoso, M.G.; Abreu, L.R.; Morais, A.R.; Guimarães, L.G.L.; Salgado, A.P.S.P.

2008. Caracterização química e efeito inibitório de óleos essenciais sobre o crescimento de Taphylococcus aureus e Escherichia coli. Ciência e Agrotecnologia, 32 (3): 887-893.

Pinheiro, P.F.; Queiroz, V.T; Rondelli, V.M.; Costa, A.V.; Marcelino, T D.P.; Pratissoli, D.

2013. Insecticidal activity of citronella grass essential oil on Frankliniella schultzei and Myzus persicae. Ciência e Agrotecnologia, 37 (2): 138-144.

Prates, H.T.; Santos, J.P.; Waquil, J.M.; Fabris, J.D.; Oliveira,

A.B.; Foster, J.E.

1998. Insecticida lactivity of monoterpenes against Ryzopertha dominica (F.) and Tribolium castaneum (Herbst). Journal of Stored Products Research, 34 (1): 243-249.

Pushpanathan, T.; Jebanesan, A.; Govindarajan, M. 2008. The essential oil of Zingiber offinalis Linn (Zingiberaceae) as a mosquito larvicidal and repellent agent against the filarial vector Culex quinquefasciatus Say (Diptera: Culicidae). Parasitology Research, 102 (1): 1289-1291.
Ranasinghe, L.; Jayawardena, B.; Abeywickrama, K.

2002. Fungicidal activity of essential oils of Cinnamomum zeylanicum (L.) and Syzygium aromaticum (L.) Merret L. M. Perry against crown rot and anthracnose pathogens isolated from banana. Letters in Applied Microbiology, 35 (1): 208-211.

Santa-Cecília, L.V.C.; Santa-Cecília, F.V.; Pedroso, E.C.; Sousa, M.V.; Abreu, F.A.; Oliveira, D.F.; Carvalho, G.A.

2010. Extratos de plantas no controle de Planococcus citri (Risso, 1813) (Hemiptera: Pseudococcidae) em cafeeiro. Coffee Science, 5 (3): 283-293.

SAS Institute.

2002. User's guide, version 8.02, TS level 2MO. SAS Institute INC, Cary, NC.

Simões, C.M.O.; Schenkel, E.P.; Gosmann, G.; Mello, J.C.P; Mentz, L.A.; Petrovick, P.R.

2007. Farmacognosia: da planta ao medicamento. 6. ed. UFSC/UFRGS. Porto Alegre, Brazil. 1104 p.

Tsukamoto, T.; Ishikawa, Y.; Miyazawa, M.

2005. Larvicidal and adulticidal activity of alkylphthalide derivates from rhizome of Cnidium officinale against Drosophila melanogaster. Journal of Agricultural and Food Chemistry, 53 (1): 5549- 5553.

Yang, Y.C.; Lee, H.S.; Lee, S.H.; Clark, J.M.; Ahn, Y.J.

2005. Ovicidal and adulticidal activities of Cinnamomum zeylanicum bark essential oil compounds and related compounds against Pediculus humanus capitis (Anoplura: Pediculicidae). International Journal for Parasitology, 35 (1): 1595-1600.

Yang, C.; Chang, X.; Zhang, M.; Ni, X.; Gong, G.; Yue, G.; Sun, X.; Chen, $\mathrm{H}$.

2017. Active compounds of stem bark extract from Schima superba and their molluscicidal effects on Pomacea canaliculata. Journal of Pest Science, 91 (1): 437-445.

Zacharia, J.T.

2011. Ecological effects of pesticide. In: Toytcheva M. (ed). Pesticides in the modern world-Risks and benefits. Croácia, InTech, pp. 129-142. 